\title{
Exploring relationships between problem gambling, scratch card gambling, and individual differences in thinking style
}

\author{
MADISON STANGE*, ALEXANDER C. WALKER, DEREK J. KOEHLER, JONATHAN A. FUGELSANG and MIKE J. DIXON
}

Department of Psychology, University of Waterloo, Waterloo, Canada

(Received: January 19, 2018; revised manuscript received: June 4, 2018; second revised manuscript received: October 31, 2018; accepted: November 22, 2018)

\begin{abstract}
Background and aims: Scratch cards are a popular form of lottery gambling available in many jurisdictions. However, there is a paucity of research that examines associations between individual differences in thinking style, participation in scratch card gambling, and problem gambling severity. Methods: In three studies, we sought to examine the relationships among these variables in large, online samples of participants. Participants completed the Cognitive Reflection Test (CRT), the Problem Gambling Severity Index (PGSI), the Actively Open-Minded Thinking Scale, and self-reported their frequency of scratch card gambling. Results: Throughout all three studies, specific associations were reliably established. Specifically, negative associations were observed between participants' CRT and PGSI scores, as well as between participants' CRT scores and scratch card gambling frequency. In addition, we found a positive association between problem gambling severity and scratch card gambling frequency. Finally, problem gambling severity was shown to correlate positively with participants' willingness to pay for irrelevant information in a scratch card gambling scenario. Discussion and conclusions: Overall, we observed that problem gambling severity is associated with an individuals' thinking style and scratch card gambling behavior. This study adds to the existing literature examining problem gambling, and highlights the role of thinking style in understanding gambling behavior and problematic gambling.
\end{abstract}

Keywords: scratch card gambling, problem gambling, individual differences, thinking style, Cognitive Reflection Test

\section{INTRODUCTION}

Lottery gambling is a popular and widespread gambling activity in Canada. Recent estimates suggest that $45 \%$ of Ontario adults are regular lottery players (Ontario Lottery and Gaming Corporation [OLG], 2016). Lottery games, as a discrete category of gambling activities, include traditional lottery (or "lotto") draws in which combinations of winning numbers are drawn daily or weekly, instant ticket or scratch card gambling, "watch "n win" terminal games, and sports games. In Canada, lottery games are available at many retailers (e.g., grocery stores, pharmacies, convenience stores, gas stations, etc.) and are typically associated with a low price point. Despite their widespread popularity and accessibility, certain types of lottery games (e.g., scratch cards) remain relatively understudied in the literature compared to other forms of gambling.

Although they are grouped together within the general category of lottery products, there is a considerable amount of heterogeneity in terms of structural characteristics within this group of products, particularly with regard to their potential for abuse or misuse. While a relatively high percentage of people report playing traditional lottery games (Ariyabuddhiphongs, 2011; Rogers, 1998), the potential for harm related to these draws is considered to be low
(Subramaniam et al., 2016). One potential reason for this is the protracted event frequency associated with weekly lottery draws (the most common type of "draw" lottery), meaning that the time between the purchase of the lottery ticket and the outcome reveal is usually quite lengthy (e.g., on the order of days; Short, Penney, Mazmanian, \& Jamieson, 2015). On the other end of the spectrum are games such as "scratch cards" or "instant tickets," which, as their name suggests, allow for outcomes to be revealed rather quickly (e.g., on the order of minutes; Griffiths, 2002; Papoff \& Norris, 2009). Another difference between traditional lottery draws and scratch card games is their capacity for continuous play. Traditional lottery draws happen on an orderly schedule, with most draws occurring weekly or sometimes daily. However, once the draw is complete, there is no other opportunity for further play. On the contrary, scratch cards are what gambling researchers refer to as a continuous form of gambling, offering players the opportunity to continue playing after each individual game is complete (Griffiths, 2002; Papoff \& Norris, 2009). In line with these features, scratch card gambling was found to

* Corresponding author: Madison Stange; Department of Psychology, University of Waterloo, 200 University Avenue West, Waterloo, ON N2L 3G1, Canada; E-mail: mstange@uwaterloo.ca

This is an open-access article distributed under the terms of the Creative Commons Attribution-NonCommercial 4.0 International License, which permits unrestricted use, distribution, and reproduction in any medium for non-commercial purposes, provided the original author and source are credited, a link to the CC License is provided, and changes - if any - are indicated. 
predict problem gambling severity over and above demographic variables, whereas participation in traditional lottery draws was not (Short et al., 2015).

Previous research has examined the relationship between problem gambling and scratch card gambling at the population level. A recent longitudinal study of gambling behavior in Canada found that frequency of scratch card play was predictive of problem gambling over time (Williams et al., 2015). In a Dutch sample, the prevalence of potential problematic scratch card gambling was estimated to be $2.68 \%$ of the sample population (DeFuentes-Merillas, Koeter, Bethleham, Schippers, \& Van Den Brink, 2003). More recently, reports from Portugal have highlighted concern about the stable increase in scratch card sales over time, as well as with the characteristics of these games that make them difficult to control from a responsible gambling perspective (Rodrigues-Silva, 2017). Finally, case studies of pathological scratch card gamblers have been reported in the literature (Raposo-Lima, Castro, Sousa, \& Morgado, 2015). These findings, coupled with the previously discussed structural characteristics of scratch card games, suggest that the relationship between problem gambling and scratch card play warrants further exploration.

One other area of gambling research that remains understudied is the influence of individual differences in thinking style, which may play a substantial role in gambling-related cognitions and behaviors. This is demonstrated in a study of game choice by Mouneyrac et al. (2018) in which being male, possessing an analytic style of thinking, and a high need for cognition were found to predict a preference for strategic forms of gambling (e.g., poker, sports betting, and black jack), compared to non-strategic forms of gambling (e.g., scratch cards, slot machines, and lotteries). However, in this study, all forms of strategic and non-strategic gambling were grouped together, thus clouding the relationships between specific individual difference variables (such as thinking style) and specific game types (such as scratch cards). This is problematic given the aforementioned heterogeneity in structural characteristics within lottery games. One additional study has examined the relationship between thinking style and problem gambling severity, uncovering a negative relation between problem gambling severity and scores on the Actively Open-Minded Thinking Scale (AOT; Stanovich \& West, 2008), suggesting that as the endorsement of open-minded thinking decreases, problem gambling severity increases (MacLaren, Fugelsang, Harrigan, \& Dixon, 2012). Therefore, we believe that it is important to further investigate the relationships between individual differences in thinking style, problem gambling, and scratch card gambling behavior.

In this series of studies, we sought to investigate how individual differences in thinking style, as measured by the Cognitive Reflection Test (CRT; Frederick, 2005), relate to the frequency with which individuals engage in scratch card gambling and their levels of problem gambling. One may predict certain associations between these variables based on previous work. Specifically, since higher scores on the CRT reflect a more analytic style of thinking, one would expect CRT scores to negatively correlate with both scratch card gambling frequency and problem gambling severity. In addition, we predict, consistent with past research
(DeFuentes-Merillas et al., 2003; Raposo-Lima et al., 2015; Williams et al., 2015), that Problem Gambling Severity Index (PGSI) scores will correlate positively with participants' frequency of scratch card gambling. Although these associations may be predicted based on the previous work discussed, no studies have directly reported relationships between these constructs, constituting a fairly large gap in the literature. In a series of three online studies, participants completed a number of relevant individual difference measures, thus allowing us to address the present research questions. These measures were collected in the context of a larger investigation into scratch

card gambling, the results of which are reported elsewhere (Walker, Stange, Fugelsang, Koehler, \& Dixon, 2018).

\section{STUDY 1}

In Study 1, we explored the associations between individual differences in thinking style, frequency of scratch card gambling, and problem gambling in the context of a larger research project. Participants were presented with images of three scratch card games, each presented alongside an information table that displayed either a low, medium, or high amount of unclaimed prizes (i.e., prizes still available to be won; see Supplemenetary Materials). The scratch card games presented mirrored real scratch card games available for purchase in our home jurisdictation of Ontario, and thus were taken from the Ontario Lottery and Gaming Corporation website (OLG, 2018). Although unclaimed prize information feels intuitively useful, it is uninformative in the absence of the number of cards remaining in circulation. For example, a scratch card may have a single unclaimed prize, yet feature only two cards remaining, making it preferable to a card that may have 10 times as many unclaimed prizes, but features more than 100 times the number of cards remaining. In the scratch card gambling task, participants rated their likelihood of winning, perceived excitement, and made hypothetical purchases for each game without card remaining information provided. As these three judgments addressed a different research question than the present investigation, they are reported elsewhere (Walker et al., 2018). Following these judgments, participants indicated how much they would be willing to pay for unclaimed prize information for all scratch card games, as an index of how much they valued this uninformative information. Finally, participants completed the CRT (Frederick, 2005), PGSI (Ferris \& Wynne, 2001), and various demographic questions, including scratch card gambling frequency. These latter constructs were explored to assess whether individual differences in thinking style, willingness to pay (WTP) for uninformative unclaimed prize information, and scratch card gambling frequency related to participants'self-reported problem gambling levels.

\section{METHODS}

\section{Participants}

A sample of 201 participants were recruited from the online crowdsourcing platform Amazon Mechanical Turk to 
complete an online questionnaire. For all studies, our full sample was recruited prior to data analysis. Participants were recruited under the condition that they were the US residents and received approval on 95\% (or more) of their past Mechanical Turk tasks. This study took approximately 20 min to complete and participants were remunerated with $\$ 3.00$ for their participation.

\section{Measures}

Willingness to pay (WTP). Participants indicated their WTP for uninformative unclaimed prize information while completing a hypothetical gambling task. Due to the uninformative nature of unclaimed prize information, those who indicated that they would be willing to pay for this information were viewed as providing a suboptimal response. Participants were presented with three scratch card game images (each version of a game titled "100× Multiplier") and were asked: "Assuming you were going to purchase five of these scratch cards, how much money would you be willing to pay for unclaimed prize information for all three versions of $100 \times$ Multiplier?" Participants responded to this item by providing a dollar amount in a free-entry text box.

Cognitive Reflection Test (CRT). The CRT (Frederick, 2005) was designed to evaluate individuals' ability to suppress an intuitive incorrect response in favor of a deliberative correct answer. Since participants from Amazon's Mechanical Turk are often presented with CRT problems, we elected to use a modified version of the 3 -item CRT. All three items utilized the same mechanistic style as Frederick's original CRT items, but featured different numbers and objects from the original questions. It is noteworthy that the intuitive incorrect response for one of our modified CRT items in Study 1 may not have been as intuitively appealing as the original CRT item it was modeled after. This is because the intuitive incorrect response for one modified CRT item did not result in a round number (this was remedied in Studies 2 and 3). The exact items used can be found in the Supplementary Materials along with each items' correct and intuitive incorrect answers. For all CRT items, participants provided their answers in a free-entry text box.

Problem Gambling Severity Index (PGSI). The PGSI (Ferris \& Wynne, 2001) is a subset of the Canadian Problem Gambling Index and provides a reliable and valid measure of problem gambling symptomatology. Participants rated nine items addressing gambling-related harms on a scale from 0 (never) to 3 (almost always). These individual items were then summed resulting in an overall score for each participant. Scores of 0 on the PGSI indicate non-problem gambling, scores between 1 and 4 indicate low-risk gambling, scores between 5 and 7 indicate moderate-risk gambling, and scores of 8 and above are indicative of problem gambling (Currie, Hodgins, \& Casey, 2013).

Demographic questions. Participants provided their age, gender, and responded to an item regarding their frequency of scratch card gambling. The latter was based on an item in the Canadian Problem Gambling Index (Ferris \& Wynne, 2001), which assesses the frequency with which individuals engage in various forms of gambling. The item ["In the past 12 months, how many times have you played an instant scratch card game (e.g., "Cash for Life")?] included eight response options $(0,1-5,6-10,11-15,16-23$, or 24 or more times in the past 12 months, I have never played this type of game, and I prefer not to say), scaling from no involvement in scratch card gambling to frequent scratch card gambling.

\section{Procedure}

Participants were presented with three scratch card games that were identical with the exception of the unclaimed prize information provided. They judged how likely they felt they were to win and how excited they were to play each scratch card game. Next, paricipants indicated their WTP for unclaimed prize information and specified how many of each scratch card they would elect to hypothetically purchase. Finally, participants completed the demographic measures, which asked about age, gender, and scratch card gambling frequency, as well as CRT (Frederick, 2005) and PGSI (Ferris \& Wynne, 2001) items. As mentioned previously, participants completed the likelihood of winning, perceived excitement, and the hypothetical purchasing task for reasons peripheral to the current research question. In addition, one base rate neglect item was included for exploratory purposes and therefore will not be discussed further.

\section{Ethics}

The study procedures were carried out in accordance with the Tri-Council Policy Statement for the Ethical Conduct for Research Involving Humans (2nd edition). The Office of Research Ethics at the University of Waterloo approved the study. All participants provided informed consent before participating.

\section{RESULTS}

Descriptive statistics were analyzed for our sample along with all presented measures in Study 1 (Table 1). Our main analyses centered around correlating our measured variables. First, participants' CRT scores were negatively correlated with their frequency of scratch card gambling, $r(197)=-.140, p=.048$, as well as their PGSI scores, $r(197)=-.302, p<.001$. Moreover, participants' PGSI scores were related to their frequency of scratch card gambling, $r(195)=.229, p=.001$, as well as the amount they were willing to pay for unclaimed prize information, $r(197)=.143, p=.044$. No other correlations reached significance; all correlations analyzed in Study 1 can be viewed in Table 2.

\section{STUDY 2}

Study 2 provided us with the opportunity to improve our measure of thinking style by including four additional CRT items. Furthermore, modifications to the experimental task resulted in participants now being provided with the number of scratch cards remaining for each game allowing them to combine this information with unclaimed prize information in order to calculate the payback percentage (i.e., the expected rate of return) of each presented scratch card 
Table 1. Descriptive statistics

\begin{tabular}{|c|c|c|c|}
\hline Measure & Study 1 & Study 2 & Study 3 \\
\hline Age [mean $(S D)]$ & $33.20(9.0)$ & $34.42(11.33)$ & $39.92(8.60)$ \\
\hline Gender $[\%$ females $]$ & $37.3 \%$ & $44.8 \%$ & $33.5 \%$ \\
\hline \multicolumn{4}{|l|}{ Frequency of scratch card gambling $[n(\%)]$} \\
\hline Had not played & $66(33.2 \%)$ & $65(32.5 \%)$ & $59(29.5 \%)$ \\
\hline $1-5$ times & $74(37.2 \%)$ & $66(33.0 \%)$ & $74(37.0 \%)$ \\
\hline $6-10$ times & $28(14.1 \%)$ & $26(13.0 \%)$ & $22(11.0 \%)$ \\
\hline $11-15$ times & $12(6.0 \%)$ & $15(7.5 \%)$ & $19(9.5 \%)$ \\
\hline 16-24 times & $8(4.0 \%)$ & $7(3.5 \%)$ & $10(5.0 \%)$ \\
\hline 24 or more & $11(5.5 \%)$ & $21(10.5 \%)$ & $16(8.0 \%)$ \\
\hline Cognitive Reflection Test [mean $(S D)]$ & $1.9(1.17)$ & $3.86(2.25)$ & $3.61(2.18)$ \\
\hline \multicolumn{4}{|l|}{ Problem Gambling Severity Index $[n(\%)]$} \\
\hline Non-problem gambling & $125(62.8 \%)$ & $111(57.2 \%)$ & $96(49.2 \%)$ \\
\hline Low-risk gambling & $54(27.1 \%)$ & $47(24.2 \%)$ & $59(30.3 \%)$ \\
\hline Moderate-risk gambling & $7(3.5 \%)$ & $11(5.7 \%)$ & $13(6.7 \%)$ \\
\hline Problem gambling & $13(6.5 \%)$ & $25(12.9 \%)$ & $27(13.8 \%)$ \\
\hline \multicolumn{4}{|l|}{ Willingness to pay } \\
\hline Mean $(S D)$ & $56.49(412.11)$ & - & - \\
\hline Median $(S D)$ & $5.00(412.11)$ & - & - \\
\hline Payback percentage calculation $[n(\%)]$ & - & $30(14.9 \%)$ & $31(15.5 \%)$ \\
\hline Actively open-minded thinking [mean $(S D)]$ & - & - & $36.38(6.61)$ \\
\hline
\end{tabular}

Note. Descriptive statistics for all measures are presented in Studies 1-3. Categories for the frequency of scratch card gambling represent participants' self-reported scratch card gambling frequency in the past 12 months. The maximum score possible on the Cognitive Reflection Test was 3 for Study 1 and 7 for Studies 2 and 3. Willingness to pay amounts represent average dollar values provided by participants. Possible scores on the Actively Open-Minded Thinking Scale ranged from 7 to 49. SD: standard deviation.

Table 2. Study 1 correlations

\begin{tabular}{lcccc}
\hline & 1 & 2 & 3 & 4 \\
\hline 1. CRT & - & & & \\
2. Scratch card frequency & $-.14^{*}$ & - & & \\
3. PGSI & $-.30^{* * *}$ & $.23^{* *}$ & - & \\
4. WTP & -.02 & -.08 & $.14^{*}$ & - \\
\hline
\end{tabular}

Note. Pearson's correlations (Study 1; $N=201$ ). CRT: Cognitive Reflection Test; PGSI: Problem Gambling Severity Index; WTP: willingness to pay.

${ }^{* * *} p<.001 .{ }^{* *} p<.01 .{ }^{*} p<.05$.

game. Importantly, this change allowed us to more easily assess participants' ability to calculate the payback percentage of a scratch card game. Differences in the ability to calculate payback percentage may relate to problem gambling severity and scratch card gambling frequency, as an understanding of this concept may influence participants' willingness to engage in gambling behaviors. One may also expect that CRT performance may relate to participants' ability to calculate payback percentage, as both require some level of mathematical ability. Finally, we sought to replicate the observed relations from Study 1.

\section{METHODS}

\section{Participants}

A sample of 201 participants were recruited from Amazon Mechanical Turk and were remunerated with $\$ 4.00$ upon completion of a 25-min online questionnaire.
All participants were recruited under the condition that they were the US residents and had a 95\% (or greater) task approval rate on Mechanical Turk.

\section{Measures}

All measures were identical to those described in Study 1 with the following exceptions presented below.

Willingness to Pay (WTP). Due to changes in the experimental task (i.e., the inclusion of scratch cards remaining information), unclaimed prize information was no longer uninformative in Studies 2 and 3. Therefore, we could no longer view those participants who indicated that they would be willing to pay for this information as providing a suboptimal response. As such, correlations involving WTP were not analyzed in Studies 2 and 3.

Cognitive Reflection Test (CRT). In Study 2, we expanded our CRT measure to feature seven items. This change was made in order to attempt to increase the variability of participants' CRT scores relative to those obtained from participants in Study 1. In addition, we believe that one reason for the high CRT scores observed in Study 1 was participants' familiarity with our CRT items. Thus, in order to combat this issue, we included four lesser-known CRT items into our scale (Toplak, West, \& Stanovich, 2014). The full list of CRT items used in Study 2 can be found in the Supplementary Materials.

Calculating payback percentage. Unlike in Study 1, the experimental task in Study 2 provided participants with all of the information necessary to calculate the payback percentage of each hypothetical scratch card game (allowing participants to optimize their scratch card choices). Thus, unlike in Study 1, we asked participants 
to demonstrate their ability to properly calculate the payback percentage of a scratch card game. Prior to asking participants to perform this calculation, participants were presented with a paragraph that explained what a payback percentage is in the context of scratch card gambling. Next, participants were asked if they would be able to properly calculate the payback percentage of a scratch card game, given that they had all the necessary information. If they responded affirmatively, participants were given all relevant information and asked to calculate the correct payback percentage of a scratch card game. Those who attempted this calculation provided their answer in a free-entry text box.

\section{Procedure}

Study 2 followed an identical procedure to that described in Study 1, with the addition of an item assessing participants' ability to correctly calculate payback percentage following the experimental task.

\section{RESULTS}

Of the 201 participants who completed Study 2, 20 participants had previously participated in Study 1 . All analyses were conducted with the non-naive participants both removed and retained; however, our results were not impacted by the presence of the non-naive participants (i.e., the interpretation of all significance tests were the same with non-naive participants removed), and therefore we retained our full sample. Sample characteristics and descriptive statistics for all presented measures in Study 2 can be found in Table 1 .

As described in Study 1, our main analysis consisted of correlating all measured variables. As predicted, CRT scores were shown to relate to participants' frequency of scratch card gambling, $r(198)=-.248, p<.001$, and PGSI scores, $r(192)=-.213, p=.003$. Furthermore, we again observed a significant positive correlation between participants' PGSI scores and the frequency with which participants took part in scratch card gambling, $r(191)=.286, p<.001$. Finally, CRT scores were shown to be associated with participants' ability to correctly calculate payback percentage, $r(199)=.350, p<.001$. No other correlations reached significance; all correlations analyzed in Study 2 can be viewed in Table 3.

Table 3. Study 2 correlations

\begin{tabular}{lcccc}
\hline & 1 & 2 & 3 & 4 \\
\hline 1. CRT & - & & & \\
2. Scratch card frequency & $-.25 * * *$ & - & & \\
3. PGSI & $-.21 * *$ & $.29 * * *$ & - & \\
4. PBP calculation & $.35^{* * *}$ & -.09 & -.11 & - \\
\hline
\end{tabular}

Note. Pearson's correlations (Study 2; $N=201$ ); replicated findings are represented in bold. CRT: Cognitive Reflection Test; PGSI: Problem Gambling Severity Index; PBP: payback percentage. $* * * p<.001 . * * p<.01$.

\section{STUDY 3}

Study 3 provided us with the opportunity to extend the results of Studies 1 and 2 by including a self-report measure of thinking style, the AOT (Haran, Ritov, \& Mellers, 2013). The AOT is a self-report measure of participants' preference for open-minded thinking, distinguishing it from a performance-based measure like the CRT. Therefore, Study 3 allowed us to measure the kinds of thinking participants value versus the types of thinking they engage in. We predicted that participants' AOT scores would correlate positively with CRT scores (as demonstrated in past research; Toplak, West, \& Stanovich, 2011), as well as correlate negatively with PGSI scores and frequency of scratch card gambling. Finally, we sought to once again replicate the statistically significant associations observed in Studies 1 and 2 .

\section{METHODS}

\section{Participants}

A sample of 200 participants were recruited from Amazon Mechanical Turk and were remunerated with $\$ 4.00$ upon completion of a 25-min online questionnaire. All participants were recruited under the condition that they were the US residents and had a 95\% (or greater) task approval rate on Mechanical Turk.

\section{Measures}

All measures were identical to those used in Study 2 with the exception that Study 3 featured the addition of the 7-item AOT scale (Haran et al., 2013).

Actively Open-Minded Thinking Scale (AOT). The AOT (Baron, 1993; Haran et al., 2013) was designed to assess peoples' beliefs about the benefits of open-minded thinking, with the ultimate goal of using peoples' beliefs about openminded thinking to predict thinking behaviors. In Study 3, participants were asked to complete the 7-item version of the AOT by stating their level of agreement with each AOT item on a 7-point scale ranging from 1 (completely disagree) to 7 (completely agree). The full list of AOT items used in Study 3 can be found in the Supplementary Materials.

\section{Procedure}

Study 3 followed an identical procedure to Studies 1 and 2, with the exeption that immediately prior to completing the study, participants in Study 3 completed seven AOT items designed to assess their beliefs about the benefits of openminded thinking.

\section{RESULTS}

Of the 200 participants who completed Study 3, 43 participants had previously participated in Studies 1 or 2 . All analyses were conducted with the non-naive participants removed and retained. When the results of these analyses 
differed, the analyses with non-naive participants removed were reported. Sample characteristics and descriptive statistics for all presented measures in Study 3 can be found in Table 1.

Once again, correlational analyses were conducted to determine how our various measures were associated with each other. As predicted, CRT scores were shown to relate to participants' frequency of scratch card gambling, $r(198)=-.194, p=.006$, and PGSI scores, $r(193)=$ $-.277, p<.001$. Next, we observed a significant positive correlation between participants' PGSI scores and the frequency with which participants engaged in scratch card gambling, $r(193)=.484, p<.001$. In addition, CRT scores were found to be correlated with participants' AOT scores, $r(196)=.368, p<.001$, and their ability to correctly calculate a scratch card's payback percentage, $r(198)=.326$, $p<.001$. Finally, AOT scores were shown to negatively correlate with participants' frequency of scratch card gambling, $r(196)=-.216, p=.002$, and their PGSI scores, $r(192)=-.455, p<.001$, but positively correlate with their ability to correctly calculate payback percentage, $r(196)=.163, \quad p=.022$. With non-naive participants removed, the correlation between AOT scores and scratch card gambling frequency was attenuated and became marginally significant, $r(153)=-.155, p=.054$. No other correlations reached significance; all correlations analyzed in Study 3 can be viewed in Table 4.

\section{DISCUSSION}

Across three studies, we observed a number of consistent relationships between various gambling-related and individual difference measures. These included twice-replicated negative associations between CRT and participants' frequency of scratch card gambling and PGSI scores, as well as a twice-replicated positive association between PGSI scores and participants' frequency of scratch card gambling.

In all three studies, we observed a small association (Cohen, 1988) between CRT performance and the frequency with which participants played scratch card games in their everyday lives, suggesting that the CRT explains a small amount of the variance in scratch card gambling frequency.

Table 4. Study 3 correlations

\begin{tabular}{lccccc}
\hline & 1 & 2 & 3 & 4 & 5 \\
\hline 1. CRT & - & & & & \\
2. Scratch card &.$- \mathbf{1 9} * *$ & - & & & \\
$\quad$ frequency & & & & & \\
3. PGSI & $-.28 * * *$ &. $\mathbf{4 8} * * *$ & - & & \\
4. PBP &. $\mathbf{3 3} * * *$ & .05 & .09 & - & \\
$\quad$ calculation & & & & & \\
5. AOT & $.37 * * *$ & $-.22 * *$ & $-.46^{* * *}$ & $.16^{*}$ & - \\
\hline
\end{tabular}

Note. Pearson's correlations (Study 3; $N=200$ ) with non-naive participants included; replicated findings are represented in bold. CRT: Cognitive Reflection Test; PGSI: Problem Gambling Severity Index; PBP: payback percentage; AOT: Actively OpenMinded Thinking Scale.

${ }^{* * *} p<.001 .{ }^{* *} p<.01 .{ }^{*} p<.05$.
The CRT is considered to measure an individual's ability to override an intuitive (but incorrect) response, in order to arrive at the correct response. Therefore, the CRT is considered to distinguish between thinking styles, such that individuals who score highly more frequently engage in analytical thinking, whereas individuals who score lower more frequently utilize intuitive thinking (Alter, Oppenheimer, Epley, \& Eyre, 2007; Frederick, 2005). Based on the small association observed, we speculate that a propensity for engaging in analytic thinking is modestly related to less frequent scratch card gambling. This finding conceptually replicates Mouneyrac et al.'s (2018) finding that analytical thinkers preferred strategic gambling games, as opposed to non-strategic forms of gambling, such as scratch cards. Furthermore, previous research has shown a relationship between CRT performance and impulsivity, demonstrating that high CRT performers are less impulsive (Frederick, 2005). Therefore, impulsivity may also play a role in the relationship between CRT and frequency of scratch card gambling, such that individuals who are impulsive may score lower on the CRT, and possibly be more inclined to impulsively purchase scratch cards in a point-of-sale context.

In a similar vein, in all three studies, we consistently observed a small to moderate relation between participants' CRT and PGSI scores, such that participants who scored lower on the CRT scored higher on the PGSI. The results obtained in this study suggest that individuals who are more likely to engage in intuitive thinking experience more gambling harm, whereas those who are more analytical in their thinking style experience less gambling harm. Previous research has also found an association between CRT performance and other measures of cognitive abilities (Frederick, 2005; Obrecht, Chapman, \& Gelman, 2009; Toplak et al., 2011). Therefore, it is possible that the relation we observed between CRT and PGSI could reflect differences in cognitive ability among those who are experiencing more gambling related harm, and vice versa (see Kaare, Mottus, \& Konstabel, 2009).

Similar to the relationship between CRT and scratch card gambling frequency, the relationship between CRT and PGSI may also be mediated by impulsivity. There is a well-established relationship between impulsivity and disordered gambling (Brevers et al., 2012; Vitaro, Arsenault, \& Tremblay, 1997). This presents the possibility that the relationship observed in the present studies may be reflective of increased impulsivity among those experiencing gambling harm and among those individuals who favor an intuitive thinking style. Future research should further examine the relationships between these variables.

We consistently observed a small to moderate positive association between problem gambling severity and frequency of scratch card purchases. Consistent with this finding, previous research has suggested that scratch card gambling is predictive of problem gambling over time (Williams et al., 2015). Therefore, although scratch cards are commonly considered to be an innocuous form of gambling, the present findings suggest that this may not be the case. Other authors have identified various structural characteristics of scratch cards that may make them more harmful for some players (Griffiths, 2002). Our observed correlations between PGSI and scratch card frequency 
support this notion. However, it remains unknown whether individuals who report more frequent scratch card purchases consequently experience more harm from their gambling, or if individuals experiencing more gambling harm seek out scratch cards more frequently. The finding that problem gamblers frequently engage in multiple forms of gambling may account for this observation (Binde, Romild, \& Volberg, 2017), suggesting that gamblers who experience more harm simply seek out many other forms of gambling. Future research should attempt to clarify the specificity and directionality of this relationship.

A small correlation between PGSI and WTP for unclaimed prize information was observed in Study 1. That is, as individuals' self-reported problem gambling severity increased, so too did their WTP increasingly large amounts for unclaimed prize information (an uninformative piece of information both in Study 1 and in the real-world context of scratch card gambling where such information is routinely made available). Relatedly, problem gamblers have been shown to be more susceptible to a range of erroneous cognitions associated with gambling games (Källmén, Andersson, \& Andren, 2008; Toneatto, 1999). This overvaluing of unclaimed prize information parallels findings within the disordered gambling literature that shows a relationship between problem gambling severity and endorsement of the gambler's fallacy (Källmén et al., 2008; Rogers, 1998), essentially overvaluing previous outcomes that have no bearing on future outcomes within a gambling scenario. It is possible that the overvaluing of uninformative information within the context of our scratch card gambling scenario reflects similar differences in information processing as is the case with other erroneous cognitions within the gambling domain. However, as always, one should be cautious when drawing conclusions on the basis of small, unreplicated correlations.

In Study 3, we included an additional individual difference measure, the AOT. The pattern of correlations observed with the AOT parallel those observed with the CRT. Specifically, similar to the CRT, participants' AOT scores showed small to moderate negative relations with problem gambling severity and scratch card gambling frequency. These findings suggest that endorsing open-minded thinking relates to gambling-relevant measures in a similar manner as analytical thinking (as measured by the CRT). In support of this idea, we found a moderate positive association between AOT and CRT scores.

\section{Limitations}

Although replicable, some of our observed correlations were small in magnitude, suggesting that we are only explaining a small percentage of variance in these cases. As such, one should exercise caution when interpreting these findings. Other associations observed were small to moderate in magnitude (Cohen, 1988), or moderate to large (Hemphill, 2003) depending on the convention used. Due to the real-world implications of the reported associations (e.g., assocations with self-perceived gambling harm), we feel that our findings have practical significance despite being small to moderate in magnitude. Furthermore, another limitation of the current investigation is that it was conducted as part of a larger research project, and thus was not the sole focus of the data collection (i.e., participants completed unrelated tasks during the study). However, the analyses reported here were exclusively conducted for the purpose of this study and are not reported elsewhere.

\section{CONCLUSIONS}

To summarize, we repeatedly observed associations between gambling-related measures, with a specific focus on scratch card gambling, and individual differences in thinking style. Specifically, we found consistent negative relations between participants' CRT scores and their frequency of scratch card gambling, as well as their problem gambling severity. Furthermore, with problem gambling severity, we observed a consistent positive relation with scratch card gambling frequency. As a whole, these results suggest associations between individual differences in thinking style, frequency of scratch card gambling behavior, and problematic gambling.

Funding sources: This research was supported by grants from the Natural Sciences and Engineering Research Council of Canada. Support for Open Access publication of this article was provided by Gambling Research Exchange Ontario (greo.ca).

Authors' contribution: MS and ACW wrote the manuscript. All authors conceived of the idea for the study and approved the design and final version of the manuscript.

Conflict of interest: The authors declare no conflict of interest.

\section{REFERENCES}

Alter, A. L., Oppenheimer, D. M., Epley, N., \& Eyre, R. N. (2007). Overcoming intuition: Metacognitive difficulty activates analytic reasoning. Journal of Experimental Psychology: General, 136(4), 569-576. doi:10.1037/0096-3445.136.4.569

Ariyabuddhiphongs, V. (2011). Lottery gambling: A review. Journal of Gambling Studies, 27(1), 15-33. doi:10.1007/ s10899-010-9194-0

Baron, J. (1993). Why teach thinking? An essay. Applied Psychology, 42(3), 191-214. doi:10.1111/j.1464-0597.1993.tb00731.x

Binde, P., Romild, U., \& Volberg, R. A. (2017). Forms of gambling, gambling involvement and problem gambling: Evidence from a Swedish population survey. International Gambling Studies, 17(3), 490-507. doi:10.1080/14459795. 2017.1360928

Brevers, D., Cleeremans, A., Verbruggen, F., Bechara, A., Kornreich, C., Verbanck, P., \& Noël, X. (2012). Impulsive action but not impulsive choice determines problem gambling severity. PLoS One, 7(11), e50647. doi:10.1371/journal.pone. 0050647 
Cohen, J. (1988). Statistical power analysis for the behavioral sciences (2nd ed.). Hillsdale, NJ: Lawrence Erlbaum Associates.

Currie, S. R., Hodgins, D. C., \& Casey, D. M. (2013). Validity of the Problem Gambling Severity Index interpretive categories. Journal of Gambling Studies, 29(2), 311-327. doi:10.1007/ s10899-012-9300-6

DeFuentes-Merillas, L., Koeter, M. W., Bethlehem, J., Schippers, G. M., \& Van Den Brink, W. (2003). Are scratchcards addictive? The prevalence of pathological scratchcard gambling among adult scratchcard buyers in the Netherlands. Addiction, 98(6), 725-731. doi:10.1046/j.1360-0443.2003.00329.x

Ferris, J. A., \& Wynne, H. J. (2001). The Canadian Problem Gambling Index. Ottawa, ON: Canadian Centre on Substance Abuse.

Frederick, S. (2005). Cognitive reflection and decision making. The Journal of Economic Perspectives, 19(4), 25-42. doi:10.1257/089533005775196732

Griffiths, M. D. (2002). Are lottery scratchcards a "hard" form of gambling? Journal of Gambling Issues, 7. doi:10.4309/jgi. 2002.7.8

Haran, U., Ritov, I., \& Mellers, B. A. (2013). The role of actively open-minded thinking in information acquisition, accuracy, and calibration. Judgment and Decision Making, 8(3), 188-201.

Hemphill, J. F. (2003). Interpreting the magnitudes of correlation coefficients. American Psychologist, 58(1), 78-79.

Kaare, P. R., Mottus, R., \& Konstabel, K. (2009). Pathological gambling in Estonia: Relationships with personality, selfesteem, emotional states and cognitive ability. Journal of Gambling Studies, 25(3), 377-390. doi:10.1007/s10899-009-9119-y

Källmén, H., Andersson, P., \& Andren, A. (2008). Are irrational beliefs and depressive mood more common among problem gamblers than non-gamblers? A survey study of Swedish problem gamblers and controls. Journal of Gambling Studies, 24(4), 441-450. doi:10.1007/s10899-008-9101-0

MacLaren, V. V., Fugelsang, J. A., Harrigan, K. A., \& Dixon, M. J. (2012). Effects of impulsivity, reinforcement sensitivity, and cognitive style on pathological gambling symptoms among frequent slot machine players. Personality and Individual Differences, 52(3), 390-394. doi:10.1016/j.paid.2011.10.044

Mouneyrac, A., Lemercier, C., Le Floch, V., Challet-Bouju, G., Moreau, A., Jacques, C., \& Giroux, I. (2018). Cognitive characteristics of strategic and non-strategic gamblers. Journal of Gambling Studies, 34(1), 199-208. doi:10.1007/s10899-0179710-6

Obrecht, N. A., Chapman, G. B., \& Gelman, R. (2009). An encounter frequency account of how experience affects likelihood estimation. Memory \& Cognition, 37(5), 632-643. doi:10.3758/MC.37.5.632

Ontario Lottery and Gaming Corporation [OLG]. (2016). OLG lottery player demographics fact sheet - 2016. Retrieved from http://www.olg.ca/assets/documents/media/lottery_player_ statistics_fact_sheet.pdf
Papoff, K. M., \& Norris, J. E. (2009). Instant ticket purchasing by Ontario baby boomers: Increasing risk for problem gamblers. Journal of Gambling Studies, 25(2), 185-199. doi:10.1007/ s10899-009-9117-0

Raposo-Lima, C., Castro, L., Sousa, N., \& Morgado, P. (2015). SCRATCH THAT! - Two case reports of scratch-card gambling disorder. Addictive Behaviors, 45, 30-33. doi:10. 1016/j.addbeh.2015.01.018

Rodrigues-Silva, N. (2017). Scratch cards in Portugal: A hidden threat. International Gambling Studies, 17(2), 332-334. doi:10.1080/14459795.2017.1317355

Rogers, P. (1998). The cognitive psychology of lottery gambling: A theoretical review. Journal of Gambling Studies, 14(2), 111-134. doi:10.1023/A:1023042708217

Short, M. M., Penney, A. M., Mazmanian, D., \& Jamieson, J. (2015). Lottery ticket and instant win ticket gambling: Exploring the distinctions. Journal of Gambling Issues, 30, 5-21. doi:10.4309/jgi.2015.30.4

Stanovich, K. E., \& West, R. F. (2008). On the relative independence of thinking biases and cognitive ability. Journal of Personality and Social Psychology, 94(4), 672-695. doi:10.1037/0022-3514.94.4.672

Subramaniam, M., Tang, B., Abdin, E., Vaingankar, J. A., Picco, L., \& Chong, S. A. (2016). Sociodemographic correlates and morbidity in lottery gamblers: Results from a population survey. Journal of Gambling Studies, 32(1), 291-305. doi:10.1007/s10899-015-9534-1

Toneatto, T. (1999). Cognitive psychopathology of problem gambling. Substance Use and Misuse, 34(11), 1593-1604. doi:10.3109/10826089909039417

Toplak, M. E., West, R. F., \& Stanovich, K. E. (2011). The Cognitive Reflection Test as a predictor of performance on heuristics-and-biases tasks. Memory \& Cognition, 39(7), 1275-1289. doi:10.3758/s13421-011-0104-1

Toplak, M. E., West, R. F., \& Stanovich, K. E. (2014). Assessing miserly information processing: An expansion of the Cognitive Reflection Test. Thinking \& Reasoning, 20(2), 147-168. doi:10.1080/13546783.2013.844729

Vitaro, F., Arsenault, L., \& Tremblay, R. E. (1997). Dispositional predictors of problem gambling in male adolescents. American Journal of Psychiatry, 154(12), 1769-1770. doi:10.1176/ ajp.154.12.1769

Walker, A. C., Stange, M., Fugelsang, J. A., Koehler, D. J., \& Dixon, M. J. (2018). Unclaimed prize information biases perceptions of winning in scratch card gambling. Journal of Gambling Studies, 34(4), 1355-1375. doi:10.1007/s10899018-9770-2

Williams, R. J., Hann, R. G., Schopflocher, D., West, B., McLaughlin, P., White, N., King, K., \& Flexhaug, T. (2015, February 20). Quinte longitudinal study of gambling and problem gambling. Report prepared for the Ontario Problem Gambling Research Centre, Guelph, ON. Retrieved from http://hdl.handle.net/10133/3641 